\title{
New Advances in Nanophotonic Device Physics
}

\author{
Richard Osgood, Jr, Jerry Dadap, Asif Ahmed and Xiang Meng \\ Department of Electrical Engineering, Columbia University \\ New York, NY USA \\ osgood@columbia.edu
}

\begin{abstract}
Advanced silicon and plasmonic nanophotonics is undergoing rapid progress due to its manifold applications in high data communication links and other applications in imaging and sensing. Our group has been at the forefront of new devices and device physics. In this talk we will first review progress in our group in a wide variety of fundamental technologies and physics needed to extend the advances in nanophotonics. We will then illustrate these ideas with several new devices types that we have recently demonstrated at Columbia based on new simulation modalities. Our approach then to modeling and simulation is to use fully accurate methods and techniques and to achieve new capabilities based on massively parallel and high-performance computation. Much of our advances are based on new hardware strengths and testing with distributed and parallel systems.
\end{abstract}

\section{Keywords}

Silicon photonics, nanomaterials, nanophotonics, integrated photonics, parallel computing and simulations.

\section{INTRODUCTION}

Rigorous computational design of optical nanostructures is an essential step in rationale development of optimized designs for emerging applications in optical communications. In fact the increasing availability of powerful computer cluster systems allows the 3D electromagnetic design needed for accurate calculation of many key optical communication devices. At the same time, advanced photonic/plasmonic devices have emerged, which are based on new materials paradigms, such as nanoscale crystals with monolayer thickness or 3D structures far less than an optical wavelength. This area includes devices of graphene, $\mathrm{MoS}_{2}$, and nanodiamond. Such nanoscale materials, utilizing the resulting new physics and design space, offer not

Permission to make digital or hard copies of all or part of this work for personal or classroom use is granted without fee provided that copies are not made or distributed for profit or commercial advantage and that copies bear this notice and the full citation on the first page. Copyrights for components of this work owned by others than ACM must be honored. Abstracting with credit is permitted. To copy otherwise, or republish, to post on servers or to redistribute to lists, requires prior specific permission and/or a fee. Request permissions from permissions@ acm.org.

NANOCOM'16, September 28-30, 2016, New York, NY, USA

(c) 2016 ACM. ISBN 978-1-4503-4061-8/16/09.. $\$ 15.00$

DOI: http://dx.dei.org/10.1145/2967446.2967469 only opportunities, but also major challenges in achieving high performance. In principle, ab-initio modelling technique and numerical simulation enables the study of such complex systems and natural phenomena. However, the quest for higher levels of details and realism in such 3D simulations requires large computational capacity and material complexity. Thus, the use of $a b$ initio modelling/numerical analysis based on in expensive large-scale parallel computing and employing cluster computing systems has become essential. In this talk, we will illustrate this capability by considering the results of computation on structures ranging from van der Waals (vdW) heterostructures nanomateirals [1,2] to plasmonic emitters for dye emission enhancement [3] to plasmonic nano light emitters [4] (see Fig.1) and nonlocal/nonlinear properties of plasmonic/silicon waveguide [5-7]. Each of these structures benefits from the use of rigorous computational approaches such as density functional theory (DFT), finite element methods (FEM), and finitedifference time-domain (FDTD) methods.
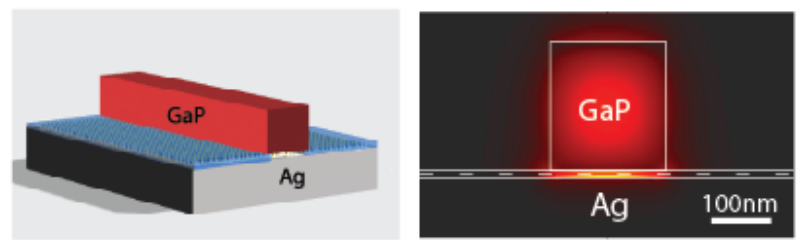

Fig. 1: (Left) Perspective view of the structure of the proposed nanolaser using a monolayer semiconductor materials as active medium. (Right) Hybrid mode profile of the nanolaser.

\section{COMPUTATION IN NANOPHOTONICS}

Our advances are possible by employing the most advanced simulation tools - taking advantage of rapidly advancing small computer technology, using new optical physics and modalities for device operation, and developing new materials approaches. Due to its importance in providing insight into the needed physics and design theory, accurate simulation places a key role in discovering new device ideas. These advances are the product of both computational algorithm and computation hardware.

\subsection{Nanomaterials Science}

Monolayer materials provide a new possible solution for future integrated photonics, due to their atomic scale thickness and the fact that this thickness allows one to "tune" electronic structure. Applications thus fact have considered new approaches to ultrafast modulation, high-speed photo-detection for optical communication, and ultracompact laser source [4]. One of the most attractive classes of these monolayer materials is that of van der Waals heterostructures; because their material tunability that have recently emerged as a novel class of nanomaterials. Electronic structural tunability for these materials is achieved by 
the spacing of their two-dimensional (2D) atomic planes or their relative in plane orientation, which give rise to new structural, and electronic properties. These artificial heterostructures can be designed and assembled by stacking individual 2D layers without the crystal-structure constraints in the usual integrated photonics devices. These in turn can lead to devices with small footprint and low power consumption. In one example, the combination of graphene's ultrahigh carrier mobility and $\mathrm{MoS}_{2}$ 's wide direct bandgap can provide a potentially unique route to high performance electronic devices. In our work here, we demonstrated using a comprehensive treatment of the electronic band structure of vdW heterostructures, via advanced DFT calculations, in conjunction with rigorous 3D electromagnetic simulation to accurately model the laser cavity and its bandgap structure (see below).

\subsection{Plasmonic Applications}

A second exciting research area in nanophotonics is that based on surface plasmon-polaritons. These electromagnetic waves propagate along metal-dielectric interfaces and can be guided by metallic nanostructures beyond the diffraction limit. The later remarkable capability has unique prospects of allowing connection between the nano (atomic) and submicrometer scale in integrated sensor systems or in Q-bit devices. As an example, we here demonstrate the computational powers to investigate the fluorescence enhancement of a molecule placed in a variable gap of a plasmonic dimer, with different dye molecules as well as different nanoparticle geometries, using a fully 3D-FDTD method. Thus in conjunction with analytic guidance based on temporal coupled-mode (TCM) theory, we show the design procedure for antennae assemblies that is useful both for general understanding of molecule-metal structure interaction and experimental efforts in plasmon-enhanced single photon source such as nitrogen vacancy center in nanodiamond crystals [8], which are important in quantum communications. In addition, as a second example of plasmonic computation, we use the plasmonic excitation process for the optically pumped nanophotonic integrated 2D laser (mentioned above). In this laser, a gap-surface-plasmon optical mode is used to achieve subwavelength optical confinement. The laser consists of a highindex GaP nanowire on a monolayer $\mathrm{MoS}_{2}$ film on a silver substrate. The optical field and materials medium are analyzed using a 3D-FDTD method, FEM and a first-principles calculation based on DFT, respectively. The realization of an ultra-compact subwavelength on-chip laser continues to be an unmet challenge for microdevice applications and optical communications; in such a laser, fundamental nonlocal E\&M physics also play an important role.

\subsection{Silicon Nanophotonics}

The field of nonlinear optics of silicon photonics has developed rapidly over the past few decades. Among the most important applications of nonlinear optics in Si photonics is the generation of light sources having different wavelengths, e.g., four wave mixing. In this application, the Si devices usually have a wide bandwidth of operation but are limited in performance due to stringent phase-matching conditions between the different frequency components. In order to overcome this obstacle, several novel techniques have been proposed among which engineering the non-Hermitian properties of the parametric amplifiers has gained much attention recently. The introduction of parity-time symmetry for four-wave mixing can lead to efficient parametric amplification. This approach has been previously demonstrated by our Group, in collaboration with Prof El_Ganainy, for optical parametric amplification in second order nonlinear material. Here we investigate this approach for third order nonlinear generation in an SOI-interconnect waveguide with nanoscale width and height using a variety of numerical simulation tools. We use dispersion engineering to obtain a waveguide geometry that can achieve proper values for second and fourth order dispersion coefficients, which in turn, are essential for perfect phase matching among the pump, signal and idler components in a degenerate four wave mixing. These devices are important for wavelength conversion or to generated mid-IR wavelengths for interconnect applications. To verify the result and gain insight into the nonlinear optical physics, we investigate the dynamical evolution of all three components under the relevant initial conditions in both undepleted and depleted pump conditions using Runge-Kutta iterative method in temporal discretization for the approximate solutions of the coupled mode equations describing the components. We find that the insertion of a loss coefficient for the idler can allow for signal amplification even when the phase matching condition is not satisfied. Our current research is to use finite difference time domain technique to more precisely understand the physical limitation in our approach.

\section{ACKNOWLEDGMENTS}

The computations were performed using the computer cluster at the Center for Functional Nanomaterials (CFN) at the Brookhaven national Laboratory (BNL), which is supported by the U.S. Department of Energy, Office of Basic Energy Sciences, under Contract No. DEAC02-98CH10886. This project is also supported by the Columbia IGERT program, outside collaboration program component (Profs. X. P. Liu, R. El-Ganainy, and N. C. Panoiu).

\section{REFERENCES}

[1] Jin, W., et al. "Tuning the electronic structure of monolayer graphene/MoS ${ }_{2}$ van der Waals heterostructures via interlayer twist."Physical Review B 92.20 (2015): 201409.

[2] Li, Y., et al. "Graphene plasmon enhanced vibrational sensing of surface-adsorbed layers." Nano Letters 14.3 (2014): 1573-1577.

[3] Meng, X, et al. "Engineering metal-nanoantennae/dye complexes for maximum fluorescence enhancement." Optics Express 22.18 (2014): 22018-22030.

[4] Meng, X., et al. "A rigorous theoretical analysis of a surfaceplasmon nanolaser with monolayer $\mathrm{MoS}_{2}$ gain medium." Optics Letters (Accepted)

[5] Khurgin, J. B. "Ultimate limit of field confinement by surface plasmon polaritons." Faraday discussions 178 (2015): 109-122.

[6] Meng, X., et al. "Dispersion Engineering of Silicon/Plasmonics Hybrid Optical Interconnections." Integrated Photonics Research, Silicon and Nanophotonics. Optical Society of America, 2015.

[7] El-Ganainy, R., Dadap, J. I., and Osgood, R.M. "Optical parametric amplification via non-Hermitian phase matching." Optics Letters 40.21 (2015): 5086-5089.

[8] Meng, X., et al. "Photoluminescence Enhancement from a Single Nitrogen Vacancy Center in a Nanodiamond Crystal via a Metal Nanoantenna." Laser Science. Optical Society of America, 2013. 